\title{
Analysis of Spun Yarn Structure of Crimped Fiber Assemblies
}

\author{
Part 2: How to Measure Phase Difference between Crimp Waves \\ By Shinji Yamaguchi* and Sueo Kawabata**, Members, TMSJ \\ *Fiber Research Laboratories, Kuraray Co., Ltd. Kurashiki, Okayama-ken \\ **Department of Polyester Chemistry, Kyoto University, Kyoto \\ Based on the Journal of the Textile Machinery Society of Japan, Transactions, Vol. 25, No. 7, T125-129 (1972)
}

\begin{abstract}
In the previous paper, the structure of helical coil assemblies was presented as a yarn model, and basing upon this structure, the distribution of the phase angles of fibers was discussed.

In this paper, a method to measure the phase angles is discribed, and it is made clear that the phase difference can be calculated by mutual correlation function $R(\tau)$ between two crimp waves. This $R(\tau)$ takes the maximum value at the position where two phase angles are coincident. The experimental results agree with the phase distributions which can be estimated from the theoretical analysis.
\end{abstract}

\section{Introduction}

In order to derive a theory on the yarn structure from the characteristics of crimped signle fibers, we presented a theory on helical coil assemblies in Part 1. Namely a yarn model was expressed by a helical coil assembly composed of many helixes having the same crimp frequency and the same radius, each touching with each other with a delay in the phase angle, but parallel with the axis of each coil.

We supposed two cases for the phase difference of two helical coils represented with $\theta$ : the one is when $\theta$ 's are random variables uniformly distributed between $0-2 \pi$, and the other exponentially distributed. With such assumptions, we decided the structure of helical coils and analyzed the relation between the observed yarn diameter and its distribution nemerically. Wishing to confirm the certainty of the assumption, we deduced the calculation method of the phase difference of crimped fibers with real yarns and then examined experimentally the relation between the crimping nature and the phase difference.

\section{Method to Calculate Phase Difference by Mutual Correlation Function of Crimp Waves}

Experiments were performed with two-ply twist spun yarns made of crimped fibers in spiral form, which is near to the helical coils used as the model mentioned previously. As shown in the pictures of Part 1, the mutual position of crimps is at random comparatively in the yarn spun with fibers having many crimps per length. In the meantime, the yarn with less crimps has a tendency to be well uniformed in the phase of crimping waves. But, it is a hard work with much labor and complications to inquire correctly the mutual phase relations of the crimps of single fibers which form the yarn. A way available is to trace the cross section of the yarn which is fixed in transparent plastic moulding. It is in fact, however, not practical in such an experiment which requires statistical treatment in many respects. A mutual correlation function seems to be an adaptable method in this case, and we present following two assumptions:

Assumption 1: Out of the fibers which form a yarn cross section, the phase of fibers contacting with each other agrees with the phase difference between two arbitrary fibers, by adopting average value of respective assemblies.

Assumption 2: The crimped fiber in space arrangement moves to get projection, changing the position from its actual position. The helical axis turns consequently with an angle of a certain degree and such is unavoidable. However, the angle is random and has no skew.

Consider $a$ helical coil with radius $a$, and another with the phase difference of $\theta$ as in Fig. 1, which is obtainable by turning it $\theta$ around the axis.

The project of them on $x-y$ plane is shown in Fig. 2 where $\theta=\delta$. By measuring $\delta$, the phase difference is obtainable. We assumed each crimped fiber forming the yarn as a helical coil and obtained projection to get the phase difference in accordance with the assumption 1 and 2 .

A given length of the yarn was marked with colors, and some fibers colored were picked out to be projected to see the mutual correlation function in crimp waves. The forms 


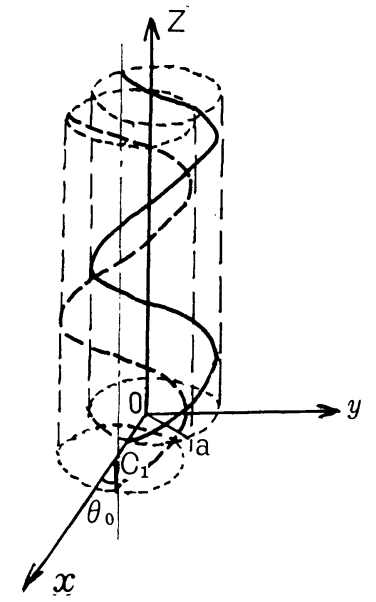

Fig. 1 Model of helical coil sover-lapped

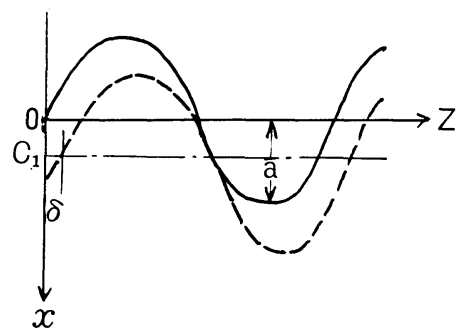

Fig. 2 Model of helical coils projected on $x-y$ plane

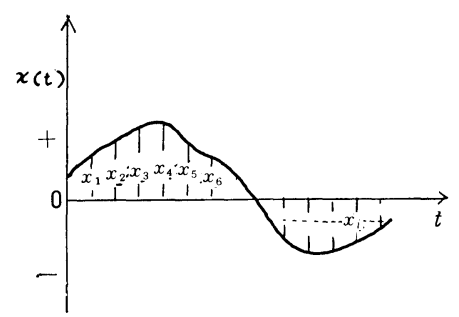

Fig. 3 Schematic representation of the 1 st crimp fiber

of crimp waves projected on the plane are shown in Figs. 3 and 4.

Let us use $x=0$ or $y=0$ for the axis of a crimped fiber.

Obtain $x(t)$ and $y(t)$ at the interval of one crimp pitch devided into nearly ten equal parts $(\Delta t=0.16 \mathrm{~mm})$. The values after this procedure are $x_{1}, x_{2}, x_{3} \ldots \ldots x_{i} \ldots \ldots, x_{n}$ and $y_{1}, y_{2}, y_{3} \ldots \ldots y_{i} \ldots \ldots y_{n}$ as shown in the figures.

The mutual correlation function between the 1 st and the 2nd crimp waves is:

$$
R_{0}=\frac{1}{N} \sum_{i-1}^{N} x_{i} y_{i}
$$

When the 2 nd is delayed by $k \Delta t(k=1,2,3)$, it is

$$
R_{k}=\frac{1}{N-k} \sum_{i=1}^{N-k} x_{i} \cdot y_{i+k}
$$

Vol. 22 No. 3 (1976)

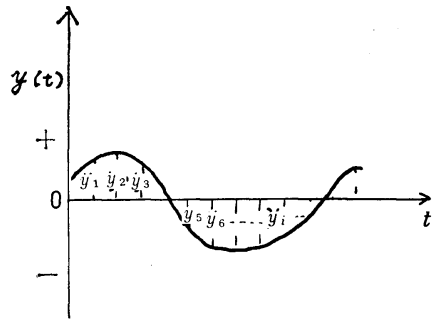

Fig. 4 Schematic representation of the 2nd crimp fiber

Take $R_{k}$ for ordinate and $k \Delta t(\equiv r)$ for abscissa. The function thus obtained is the mutual correlation function $R(\tau)$. We considered the phase would agree with $\tau$, at which position $R(\tau)$ becomes the largest. The phase was calculated by converting the position through the average pitch. The contact point of a fiber in a yarn would be many and never be limited to one. But, in our experimental calculation, we treated in Part 1,3 fibers as a set, presenting the whole composition of fibers. In measuring the phase difference, too, we use three fibers as a set. With a set of 3 fibers selected at random, we decide two phase differences $\theta_{01}$, and $\theta_{02}$, basing on the 1st crimped fiber. Under such way, we pick up all fibers which form the yarn three by three and obtain the phase difference. In the meantime, we fix a fiber at random as a standard fiber for the experiment. The first fiber of each 3-fibers in a set is compared with the standard fiber to find out the phase differences. Thus the phase differences of all fibers are calculated and the distribution of the phase difference becomes clear.

\section{Experimental Method}

The material used for the experiment was the same lowtwist two-ply polyester spun yarn with spiral crimps as in Part 1 . The yarn was dyed for marking by pressing yarn ends with iron strips.

The number of fibers composed of the yarn amounts to 94-100. Since the fibers were irregular in length due to spinning, only 63 pcs. which covered the specimen length were picked up for the experiment. Single fibers selected from the yarn were put in a paper frame so that the marks come to be arranged in uniform. Fibers were stretched
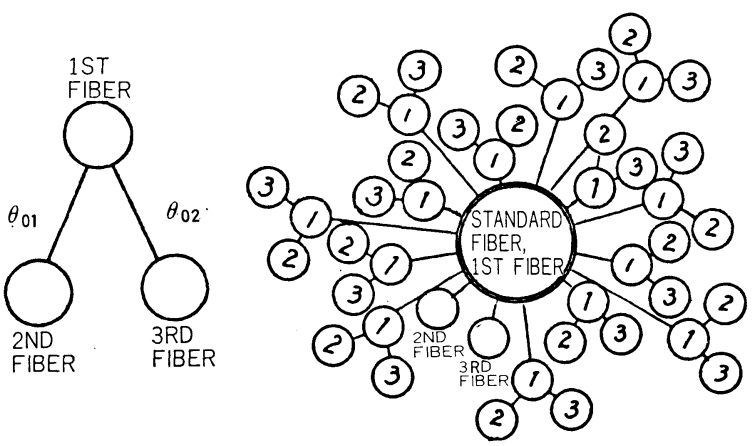

Fig. 5 Fiber assembly for calculating phase difference 


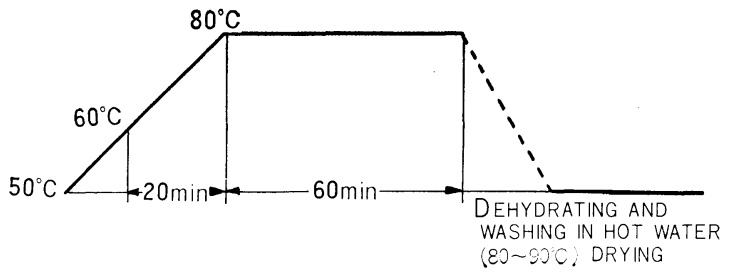

Fig. 6 Dyeing condition

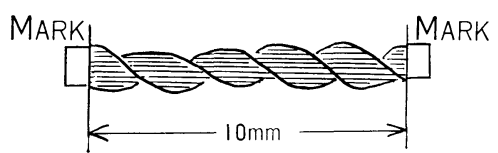

Fig. 7 Test material after being marked

$10 \%$ longer in length to eliminate twist effect. After such jobs, the fibers were fixed on the slide glass. Figs. 8 and 9 show the side views of marked yarns and single fibers fixed on the slide glass plate.

The crimp, which was fixed on the slide glass plate not to be crushed, was projected by 30 times as large and the crimp waves observed were delined. With the crimp waves such as shown in Figs. 3 and 4, we calculated mutual correlation function as follows:

$$
\begin{aligned}
& R_{k}=\frac{1}{N-k} \sum_{i=1}^{N-k} x_{i} \cdot y_{i+k} \\
& N=50 \text { (actually measured length was } 8.0 \mathrm{~mm} \text { ) } \\
& k=0 \sim 10
\end{aligned}
$$

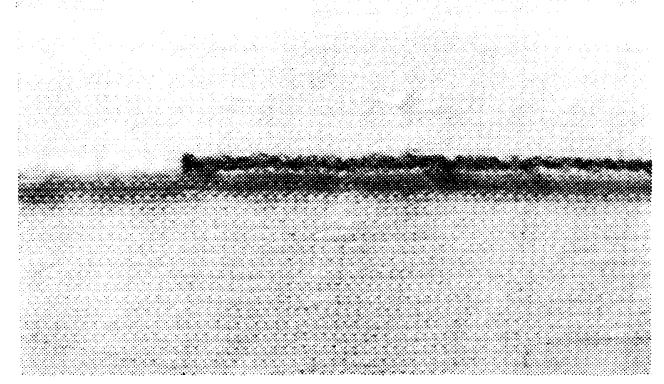

Fig. 8 Yarns dyed for marking

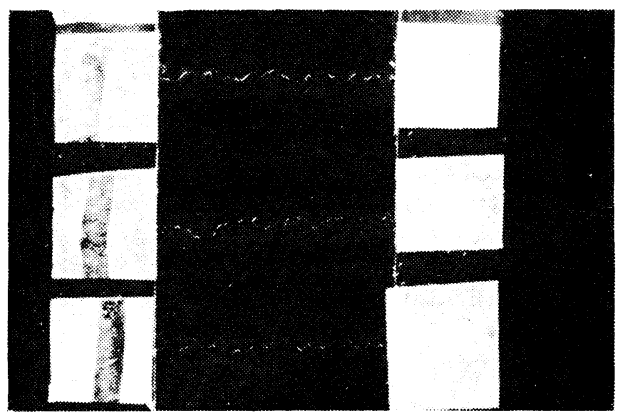

Fig. 9 Single fibers fixed on slide glass plate
As stated before, we took $R(\tau)$ and $\tau$ on ordinate and abscissa respectively, and assumed that the phases agree at the position where $R(\tau)$ becomes the largest. The phase is calculated to be $360^{\circ}$ per pitch. With the specimens used:

High crimp (22 crimps $/ 25 \mathrm{~mm}): 1$ pitch, $360^{\circ}=$ $25 / 22=1.138 \mathrm{~mm}$

1 pitch, $360^{\circ}=1.138 / 0.16=7.1 \tau$

Middle crimp $(13.9 \mathrm{crimps} / 25 \mathrm{~mm}): 1$ pitch, $360^{\circ}=$ $25 / 13.9=1.80 \mathrm{~mm}$

1 pitch, $360^{\circ}=1.80 / 0.16=11.3 \tau$

\section{Results and Consideration}

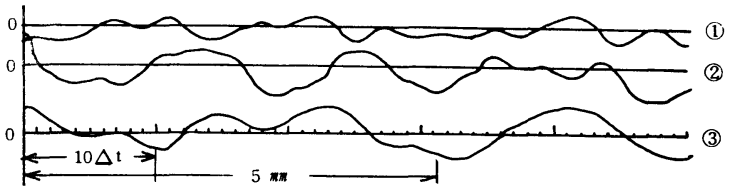

Fig. 10 An example of middle crimp

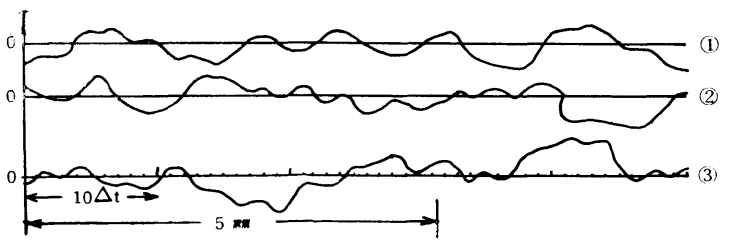

Fig. 11 An example of high crimp

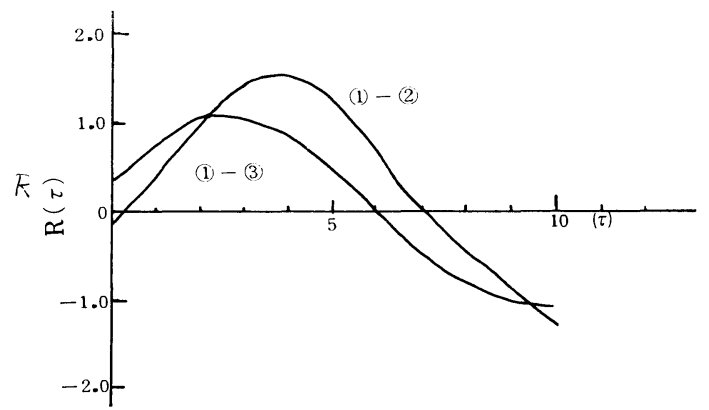

Fig. 12 An example of $R(\tau)-\tau$ of middle crimp

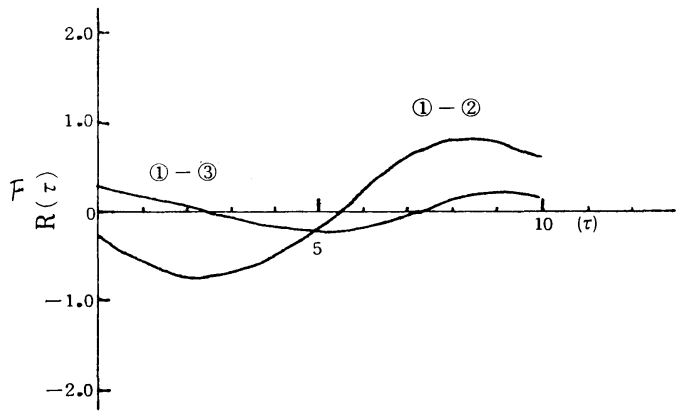

Fig. 13 An example of $R(\tau)-\tau$ of high crimp

Journal of The Textile Machinery Society of Japan 
Fig. 10 is an example of a projected middle crimp wave enlarged, and Fig. 11 another example for high crimp wave also enlarged.

The graphs of mutual correlation function of $R(\tau)-\tau$ which represents the waves in the above Fig. 10 or 11 are shown in Figs. 12 and 13.

The mutual correlation function shown in Fig. 12 or 13 changes to the curved line having the largest value with the increase of $\tau$. This means that the result already expected in Section 2 is obtainable. Considering that the phases agree at the $\tau$ position where $R(\tau)$ becomes the maximum, this position is converted in terms of averaged crimp pitch. Let us have the phase differences of all the fibers forming a yarn, and we can see the phase difference distributions as Figs. 14 and 15.

In case of high crimp, the phase difference distribution nears a random distribution. Contrarily to the above, the phase distribution in case of middle crimp has high density on the side of small phase difference, and low density on the side of large phase difference.

We show in Fig. 16 the phase difference distributing obtained in Part 1 by theoretical calculation, relying on exponentially distributed random variables, and in Fig. 17 that on uniformly distributed random variables.

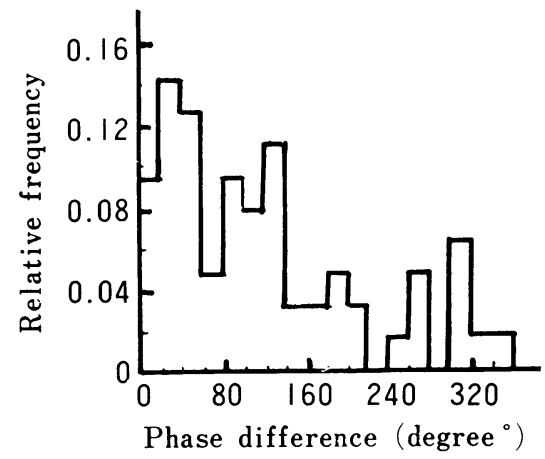

Fig. 14 Relative frequeneies of phase differences of a middle crimp yarn

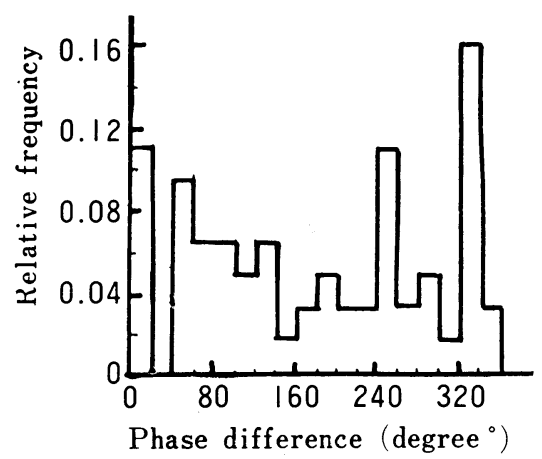

Fig. 15 Relative frequencies of phase differences of a high crimp yarn

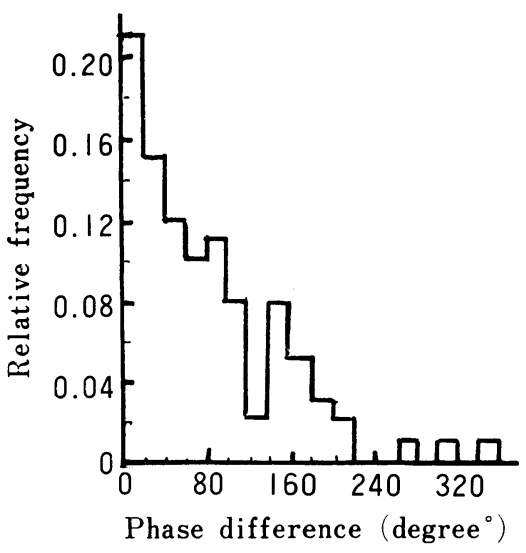

Fig. 16 Phase difference distribution when exponentially distributed random variables are used $(1 / \lambda=0.25)$

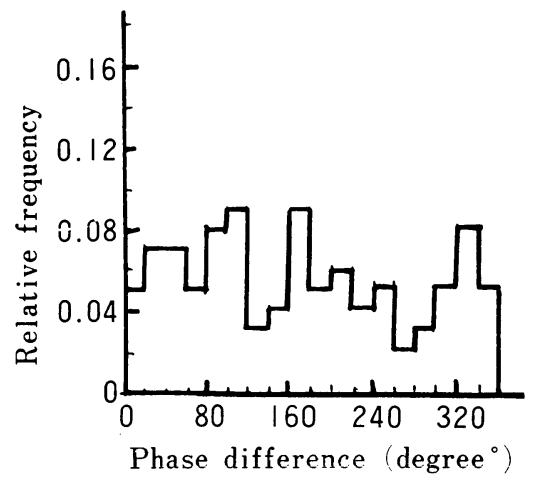

Fig. 17 Phase difference distribution when uniformly distributed random variables are used

In case of middle crimp, the phase difference distribution is closer to that due to exponentially distributed random variables, while that in case of high crimp approaches that due to uniformly distributed between $\theta=0-360^{\circ}$. This fact agrees with the result estimated by the observed yarn diameter shown in Part 1.

The delay in the phase difference of crimped fibers is an important factor to decide the yarn structure. And we regard as appropriate the theory on the structure of assembled helical coils which we adopted as an important factor for the study of the yarn structure.

Referring to Figs. 12 and 13, it becomes clear that the maximum value of the mutual correlation function is larger for middle crimp than for high crimp. Figs. 18 and 19 show the results of the comparison of $R(\tau)$ maximum in all cases:

In case of middle crimp, the maximum $R(\tau)$ is larger, but that of $R(\tau)$ in high crimp is smaller.

Our study is practiced on an assumption that the crimp pitch is always unified. The fact that the peak of $R(\tau)$ is 


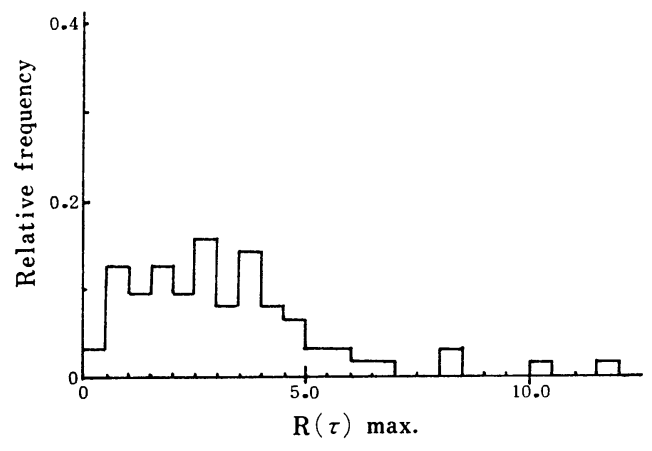

Fig. 18 Relative frequency distribution of $R(\tau)$ max. in case of middle crimp

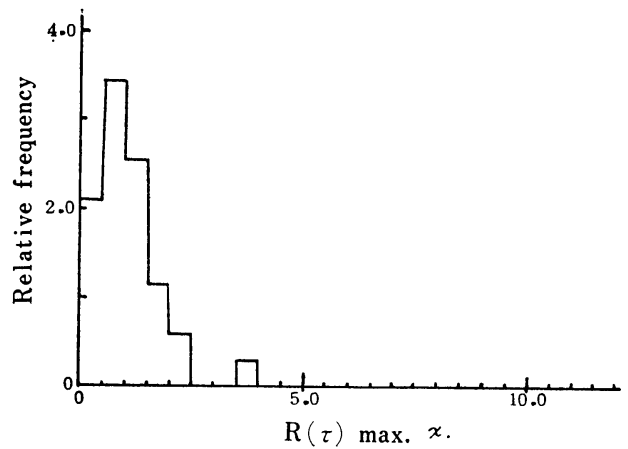

Fig. 19 Relative frequency distribution of $R(\tau)$ max. in case of high crimp

smaller means a deviation from our model structure. The statistical procedure applied in the present research includes such cases as stated above, and it seems a proper method to estimate the distribution of the phase differences. Such can be considered to be reasonable from the characteristic of the mutual correlation function. Fol'owing the increase of the crimp number, however, the deviation from the above assumption would become larger. Such would give influence upon the yarn bulkiness, for which further research is required.

\section{Conclusion}

We presented a method how to measure the delay in the phase of crimp which decides the structure of a yarn. We measured the phase differ:nces of crimp yarns, and we clarified the relationship between the nature of crimp and its phase.

1) We introduced a method to see the delay in the phase of crimp yarns by the mutual correlation function for crimp waves.

2) The delay in the phase of crimps between two fibers can be estimated by the angle when the mutual correlation function for crimp waves is at the maximum value. This almost agrees with the phase distribution calculated from the nature of yarn bulkiness. The above proves that the theory on the structure of yarn assemblies introduced in Part 1 is reasonable.

3) In case of small crimp, the delay in the phase of crimp waves decreases. But in case of high crimp, the phase delay becomes random, distributing uniformly between $\theta=$ $0-360^{\circ}$.

At the close of the present report, we express our best thanks to the members of the Fiber Assembly Study Group of Polymer Chemistry, Kyoto University, Kyoto who helped us by giving useful advices.

\section{Literatures:}

[1] Yamaguchi, Kawabata: J. Text. Mach. Soc. Japan, 25, No. 5 T83 (1972)

[2] Noroda, Kawabata, Kawai: J. Text. Mach. Soc. Japan, 22, No. 7/8 T-168 (1969) 